\title{
Coding Aspects of Secure GNSS Receivers
}

\author{
James T. Curran*, Member, IEEE, Monica Navarro ${ }^{\dagger}$, Senior Member, IEEE, Marco Anghileri ${ }^{\ddagger}$ \\ Pau Closas ${ }^{\dagger}$, Senior Member, IEEE, Stephan Pfletschinger ${ }^{\S}$ Senior Member, IEEE. \\ ${ }^{*}$ Joint Research Center of the European Commission, Ispra (VA), Italy \\ ${ }^{\dagger}$ Centre Tecnológic de Telecomunicacions de Catalunya (CTTC), Barcelona, Spain \\ ${ }^{\ddagger}$ Airbus Defence \& Space, Munich, Germany \\ $\S$ Offenburg University of Applied Sciences, Offenburg, Germany
}

\begin{abstract}
This article presents an overview of the coding aspects of a GNSS receiver. Coding allows detection and correction of channelinduced errors at the receiver, here the focus is on the mitigation of threats from malicious interferences. Although the effects of interference at different stages of GNSS baseband processing has been deeply analyzed in the literature, little attention was devoted to its impact on the navigation message decoding stage. The article provides an introduction to the various coding schemes employed by current GNSS signals, discussing their performance in the presence of noise in terms of block-error rate. Additionally, the benefits of soft-decoding schemes for navigation message decoding are highlighted when jamming interferences are present. The proposed scheme requires estimating the noise plus interference power, yielding to enhanced decoding performances under severe jamming conditions. Finally, cryptographic schemes as a means of providing anti-spoofing for geosecurity location-based services, and their potential vulnerability are discussed, with particular emphasis on the dependence on the dependence of the scheme on successful navigation message decoding.
\end{abstract}

\section{Index Terms}

bit-error rate, coding theory, Galileo, global navigation satellite systems, interference, jamming, navigation message authentication, soft-decoding, spoofing. 


\section{Coding Aspects of Secure GNSS Receivers}

\section{INTRODUCTION}

$\mathbf{S}$ ECURE navigation is a feature demanded in modern Global Navigation Satellite System (GNSS), encompassing not only reliability and integrity but also authentication of legitimate transmission. Interference signals can cause GNSS signal degradation, denial of service, or even counterfeit transmissions to control the receiver positioning solution. The effects of several types of intentional and unintentional interferences were reported in the literature [1-10] as well as some solutions. Mainly, these analyses were focused on the baseband processing of GNSS receivers, where interference countermeasures can be implemented at different stages of the receiver including the antenna design (e.g., including enhanced reception patterns and multi-antenna schemes [11-18, 1820]), radio-frequency front-end (e.g., multi-level Analogueto-Digital Converter (ADC) [21], adaptive quantization levels [22], or interference detection [23]), and signal processing (e.g. pulse blanking [24], adaptive notch filter [25]).

In contrast, the effects of interferences on the decoding performance of the receiver have not been deeply analyzed in the literature. This article provides a discussion on these effects and proposes potential solutions to improve decoding under interference. Particularly, three aspects of coding for secure GNSS receivers are examined in this work: the coding scheme applied to the navigation message at the satellite; the decoding schemes employed by the receiver; and the properties of the unreliable channel between the satellite and the user which can be counterfeit by a malicious agent.

Although early GNSS signals carried an uncoded navigation message, the benefits of error correcting codes for GNSS have been recognized and all modern GNSS signals employ forward-error correction (FEC) [26, 27].The physical channel between the satellite and the receiver plays one of the most important roles in defining reception performance. To date, the performance gain introduced by the different channel code configurations (i.e., code family, codeword length, code rate, etc.), typically measured in terms of Frame-Error Rate (FER) or block-error rate (BLER), are evaluated under rather benign cases of memory-less additive white Gaussian noise (AWGN) channels, or dynamic channels which are characterized by random burst errors [28]. However, BLER can be severely degraded in the presence of interference, especially under malicious interference or jamming.

To the best of our knowledge there is no complete characterization of the signal model from the analogue received signal down to baseband signal model at the decoder input, for the vast variety of intentional or unintentional interference types. Furthermore, little attention has been placed to the specific effect of interference in the decoding functionality, after symbol demodulation. In this work, we focus on the impact of jamming signals to channel decoding algorithms, in particular over soft-decision decoding where the accurate estimate of the noise plus interference power plays an important role. To perform this analysis, the general problem of GNSS signal demodulation and data decoding in the presence of noise and interference is formulated and provides a general description of the most representative GNSS channel codes together with baseline decoding performance.

Subsequently, robust decoding is examined in Sec. III in the context of pulsed interference. The case study assumes the interference is strong enough to degrade the decoding reliability but not sufficiently high to prevent the receiver previous stages (i.e. baseband signal processing) to be functional. In order words, it is assumed the receiver is able to provide the decoder with, potentially corrupted, demodulated symbols. The presence of the spreading sequence allows for a low complexity noise plus jammer power estimation per code symbol that significantly improves the performance of soft-decision decoding. The considered pulsed interference model, although it is not the only type of intentional interference, provides a relevant model to understand the impact of interference duration and intensity within the codeword and gain insight on possible enhancements.

Besides robustness, the presence of coding has also contributed to improvements in the integrity of the GNSS system. Where Sec. III discusses schemes to improve decoding reliability, regardless of the underlying data being genuine or not, in Sec. IV the paper examines coding schemes for anti-spoofing with an emphasis on robustness against a malicious adversary who wishes to disrupt receiver operation. The feasibility of operating such a scheme on a noisy channel is examined, and the particular influence of the the errorcorrection on the anti-spoofing schemes is assessed.

There is a rich literature regarding detection and mitigation of spoofing signals, mainly operating at signal, observables, or position levels of the receiver [29-36]. Complementarily, in this section we study the benefits that coding schemes can bring in the anti-spoofing context.

\section{A. Signal and Threat Models}

This work considers the GNSS signal in the presence of an additive thermal noise and a malicious interference. The received signal can be written as:

$$
\begin{aligned}
y(t) & =\sqrt{2 C} d(t-\tau) s(t-\tau) \sin (\omega(t) t+\theta) \\
& +w(t)+j(t),
\end{aligned}
$$

where $C$ is the received signal power in Watts, $\omega$ and $\theta$, respectively denote the frequency and phase of the received signal. The functions $d$ and $s$ respectively denote the symbols of the message content, and the direct-sequence spread spectrum (DSSS) code-division multiple access (CDMA) spreading 
sequence. Thermal noise is modeled as an AWGN function, $w(t)$ which has a one-sided spectral density of $N_{0} \mathrm{dBW} / \mathrm{Hz}$.

The malicious interference term, $j(t)$ can take on many forms, but it can be modeled as a modulated carrier:

$$
j(t)=\sqrt{2 P_{j}(t)} \sin \left(\omega_{j}(t) t\right),
$$

where the $P_{j}$ is a potentially time-varying power, for example modeling a pulsed interference; and $\omega_{j}$ is potentially timevarying frequency, for example modeling a swept interference. By choosing $P_{j}$ and $\omega_{j}$ appropriately, a broad range of interference types can be modeled.

Here, the receiver is considered as an linear time-invariant (LTI) system, which implements a maximum likelihood estimation of the received signal. In terms of data-recovery, signal is observed at the output of a matched filter, such that the observation at the end of the $n^{\text {th }}$ symbol period is given by:

$$
Y_{n}=\int_{(n-1) T_{s}}^{n T_{s}} y(t) s(t-\hat{\tau}) e^{-i(\hat{\omega} t+\hat{\theta})} d t,
$$

where $T_{s}$ represents the period of symbols of $d$, and the notation $\hat{x}$ represents the receiver's local estimate of a signal parameter $x$. For simplicity, this work considers that the receiver is perfectly synchronized with the incoming signal, such that frequency, phase and delay are perfectly matched.

One of the key features of GNSS signals which provides resilience to interference, is the DSSS modulation, which can provide a degree of spectral separation between relatively narrow-band interference signals, or other signals with significantly different spectral properties. As such, the interference contribution to $Y_{n}$ in (3) is often modeled as AWGN, where the variance is determined by the spectral separation between $j(t)$ and $s(t)$.

When assessing the performance of various coding and decoding schemes it is useful to consider a number of signal quality metrics. Traditionally, GNSS signal quality is considered in terms of the carrier power to thermal noise spectral density ratio, $C / N_{0}$, however, when dealing with the observation, $Y_{n}$, the integration period becomes important. Three different integration periods are considered here: the spreading symbol period, $T_{c}$; the encoded symbol period, $T_{s}$; and the data bit period, $T_{b}$. When decoding a received signal, these periods determine the maximum signal energy that can be observed (signal power $\times$ observation period). This metric, combined with the noise spectral density ratio, typically determines the decoding error rate.

Because the interference contribution to $Y_{n}$ can be considered AWGN, it is convenient to consider an equivalent spectral density denoted $J_{0}$. The value of $J_{0}$ is assigned based on the average value of $P_{j}$, denoted $\bar{P}_{j}$, and the spectral separation between $j(t)$ and $s(t)$, such that if an AWGN signal with a one-sided spectral density of $J_{0}$ were observed in place of $j(t)$ in (1), the statistics of $Y_{n}$ would be unchanged. This spectral separation coefficient can be found via:

$$
\operatorname{SSC}_{s, j}=\int_{-\infty}^{\infty} G_{s}(\omega) G_{j}(\omega) d \omega
$$

TABLE I

SIGNAL QUALITY METRICS FOR MESSAGE DECODING

\begin{tabular}{c|c|c|c} 
& \multirow{2}{*}{ Noise Only } & \multicolumn{2}{|c}{ Noise \& Interference } \\
& & Average & Instantaneous \\
\hline$C / N_{0}$ & $\frac{C}{N_{0}}$ & $\frac{C}{N_{0}+J_{0}}$ & $\frac{C}{N_{0}+J_{0} / \rho}$ \\
$E / N_{0}$ & $\frac{C T}{N_{0}}$ & $\frac{C T}{N_{0}+J_{0}}$ & $\frac{C T}{N_{0}+J_{0} / \rho}$ \\
$\mathrm{SNR}$ & $\frac{2 T C}{N_{0}}$ & $\frac{2 T C}{N_{0}+J_{0}}$ & $\frac{2 T C}{N_{0}+J_{0} / \rho}$
\end{tabular}

where $G_{x}(\omega)$ represents the normalized power-spectral density of $x$ [37]. Thus, the equivalent interference noise spectral density can be approximated by:

$$
J_{0}=2 \bar{P}_{j} \operatorname{SSC}_{s, j} .
$$

Interestingly, for narrow-band interference signals, SSC can be well approximated by the chip period, $T_{c}$, of the desired signal. For example, when considering a continuous wave interference and a 1.023 Mcps spreading sequence, then $\mathrm{SSC}_{s, j} \approx-60 \mathrm{~dB}$.

The rationale for considering the average power in (5) is that the interference may be observed intermittently by the receiver. For example, many interference sources take the form of continuous wave signal which is swept rapidly across a wide frequency range, exceeding the receive bandwidth of the affected receiver. From the perspective of the receiver the interference is intermittently present. Similarly, an interference source may remain within the bandwidth of a receiver, but may exhibit a pulsed amplitude modulation. When the interference repetition period is short with respect to $T_{s}$, the net effect is that of a continuously visible interference of reduced power. Specifically, if the interference signal is visible to a receiver for a fraction, $\rho$, of the time, then $\bar{P}_{j}=\rho P_{j}$, and the equivalent average one-sided spectral density of the AWGN experienced by the receiver is given by: $N_{0}+J_{0}$. In this case $\rho$ might be equal to the duty cycle of a pulsed interference, or the fraction of time a swept interference remains in-band. In the work that follows, it is assumed that $J_{0}$ can be found for a given interference signal via (4) and (5).

In contrast, when the received signal is observed over a period which is very short relative to the pulse or sweep period, then the instantaneous equivalent AWGN spectral density must be considered, which is given by $N_{0}$ in the absence of interference, and by $N_{0}+J_{0} / \rho$ in its presence. Considering the above, a number of useful signal quality metrics can be defined, for a given observation period, $T$.

These various definitions are presented in Tab. I which links the average and instantaneous values of the $C / N_{0}, E / N_{0}$, and SNR both in the presence and absence of pulsed interference. The period, $T$, can represent any of $T_{c}, T_{s}$ or $T_{b}$.

\section{CODING SCHEMES FOR GNSS}

Like other wireless communication signals, navigation signals are subject to noise, multipath and shadowing effects which may induce errors in the received data. Modern navigation signals employ some techniques to detect and correct these errors. Generally speaking, error correction may be realized in two different ways. The first is called automatic repeat 
TABLE II

CODING SCHEMES USED IN GNSS SIGNALS

\begin{tabular}{cl|c|c} 
System & Signal & Message & Coding \\
\hline GPS & L1 C/A & LNAV & none \\
& L1C & CNAV-2 & block: BCH \& LDPC \\
& L2C & CNAV & $1 / 2$-rate convolutional \\
& L5 & CNAV & $1 / 2$-rate convolutional \\
\hline Galileo & E1-B & INAV & $1 / 2-$ rate convolutional \\
& E6-B & CNAV & $1 / 2$-rate convolutional \\
& E5a & FNAV & $1 / 2$-rate convolutional \\
& E5b & INAV & $1 / 2$-rate convolutional \\
\hline SBAS & L1 & SBAS & $1 / 2$-rate convolutional
\end{tabular}

request (ARQ), sometimes also referred to as backward error correction, whereby every block of data received is checked using an error detection code: if the check fails, retransmission of the data is requested. However, since navigation systems are one-way, or simplex, systems FEC techniques are used, whereby the sender encodes the data using an error-correcting code prior to transmission. The additional information added by the code, called also redundancy, is used by the receiver to recover the original data.

Galileo, modernized GPS as well as space-based augmentation systems (SBAS) (e.g. WAAS, EGNOS) messages make use of FEC. In the following the various error protection techniques used by these systems are described and some details are given for the encoding and decoding processes.

The fundamental principle of channel coding is to add redundancy to the navigation message, which is used by a receiver to detect or correct possible errors in the received symbols. The redundant bits added by the encoder are a function of the original information. The original bits may or may not be directly visible in the encoded message. In the first case the encoding is called systematic while in the second case it is called non-systematic.

Channel coding techniques can be further divided into block codes and convolutional codes. Block codes operate on fixedsized blocks of data, each of which are encoded separately, while convolution codes operate on a continuous stream of input data. Both kinds of codes are employed in GNSS, a few of which are detailed in Table II.

\section{A. Convolutional Codes}

Binary, $1 / r$-rate, convolutional encoding, as used in GNSS, can be considered as a discrete-time, single-input, $r$-output finite impulse response (FIR) LTI system operating over the finite field, $\mathbb{F}_{2}$. Particular encoding schemes can be parametrized by the following: encoding rate, $r$, denoting the number of output bits generated for each input bit; the set of $r$ impulse responses, $G_{i}$; the constraint length, $L$, equal to the length of the longest impulse response.

The encoder operates by convolving the input sequence with each of the $r$ impulse responses, $G_{i}=$ $\left\{g_{i}(0), g_{i}(1), \ldots, g_{i}(L-1)\right\}$, to produce $r$ binary sequences. Basic $1 / r$-rate encoders interleave the bits of these $r$ sequences to form one output stream. Similar, $k / r$-rate codes, often referred to as punctured or perforated codes, can be generated from their corresponding $1 / r$ encoder by applying a puncturing matrix, which implements a deterministic omission of

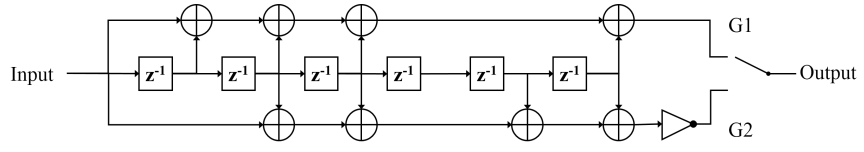

Fig. 1. A block diagram of the 1/2-rate, convolutional encoder with a constraint length of 7 , as used by Galileo having $\mathrm{G}_{1}=171 \mathrm{o}$ and $G_{2}=133 \mathrm{o}$.

certain output bits [38]. As an example, Fig. 1 depicts the convolutional encoder used by Galileo, where $z^{-1}$ denotes a unit delay.

At the receiver, the most common method of parsing the received symbols is via the use of a Viterbi decoder [39], which offers near-optimal decoding in many cases [40]. This dynamic programming technique finds the most likely sequence of original navigation bits in the sequence of received symbols. It operates by examining the difference between each received symbol and all possible symbols in the alphabet, known as the branch metric, summed across the sequence of received symbols, known as the path metric. The most likely sequence of transmitted bits is that which corresponds to the lowest path metric. Implementations of the decoder can differ in how the branch metric is computed, and can implement hard-decoding, where the branch metric is computed as the Hamming-distance between received symbols and symbols in the alphabet; or as soft-decoding, where each received symbol may be ascribed a certain weighting when computing the branch metric.

The coding operation can be applied to a continuous stream of bits, or can be applied block-wise. Galileo, for example, applies the coding block-wise, wherein each page of the navigation message is encoded separately. In this way the initial state of the encoder is always '000000', moreover, a specific set of tail bits are included to force the encoder to return to this state at the end of each block. Each block is separated by a predefined synchronization word to identify the beginning of each encoded page. In contrast, the encoding of the CNAV message on L2C and L5, as well as EGNOS and WAAS, is done as a continuous stream.

\section{B. BCH Codes}

As different parts of a GNSS message may have different importance, or may need to be extracted in isolation from other parts of the message, it can be valuable to implement a separate encoding of certain sequences of bits. In the case of GPS L1C, the CNAV-2 message will employ a separate encoding scheme on one of its subframes, containing the time-of-interval (TOI) counter, a parameter that a receiver may require to access rapidly and reliably.

A cyclic error correcting code, known as a Bose-ChaudhuriHocquenghem $(\mathrm{BCH})$ code is used in a configuration which provides very high redundancy and, thereby, very high errorcorrection capability. Specifically, a $\operatorname{BCH}(51,9)$ encoding is used, which produces 51 output symbols for every 9 data bits. Exact encoding and decoding procedure can be found, for example, in [41]. One simple implementation of the encoder is that of a linear feedback shift register (LFSR), as depicted in Fig. 2. 


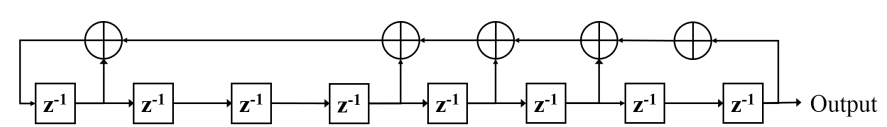

Fig. 2. An example shift-register implementation of a $\mathrm{BCH}$ encoder for the polynomial: $1+x+x^{4}+x^{5}+x^{6}+x^{7}+x^{8}$.

Although many algorithms exist for $\mathrm{BCH}$ decoding, due to the extremely high redundancy of this particular implementation, it is relatively easy for a receiver to simply implement a brute-force check of all $2^{9}=512$ possible messages, comparing the locally generated codeword against that which is received. The comparison may be implemented as a harddecoding, taking the Hamming-distance between generated and received symbols, or may implement soft-decoding and effect a maximum-likelihood (ML) decoder.

\section{LDPC Codes}

Binary low-density parity-check (LDPC) codes are a class of systematic, linear error-correcting codes defined as the null space of a binary $(n-k) \times n$ parity-check matrix $\mathbf{H}$, where $k$ and $n$ indicate the length of the information part and of the codeword respectively [42]. With a low density parity check matrix, $\mathbf{H}$, these codes can achieve spectral efficiencies close to that of the channel capacity [43, 44]. Regular LDPC codes are characterized by a parity-check matrix, where columns and rows exhibit constant Hamming weights and equal density, while irregular LDPC codes, which exhibit slightly better performance, have non-constant column and row Hamming weights $[44,45]$. An irregular LDPC code has been employed for the GPS L1C signal for all of the navigation data, other than the TOI [41].

Central to the definition of a particular LDPC code is the parity check matrix, H. Details of the GPS L1C, for example, can be found in [41].

Typically, however, the matrix is constructed from a set of sub-matrices, [41, 44] as shown in (6). Note that the dimensions of the matrix $\mathbf{H}$ are specific to the length of the data to be encoded. For the GPS L1C message, two $\mathbf{H}$ matrices are defined, one for the second subframe, having dimension $600 \times 1200$, and another for the third subframe, having dimensions $274 \times 548$. Both versions of the LDPC codes produce a coding rate of $1 / 2$.

$$
\mathbf{H}=\left(\begin{array}{c|c|c} 
& & \\
\mathbf{A} & \mathbf{B} & \mathbf{T} \\
& & \\
\hline \mathbf{C} & \mathbf{D} & \mathbf{E}
\end{array}\right)
$$

The encoded message contains systematic portion, containing the original data bits $u$, and two parity check portions $\mathbf{p}_{1}$ and

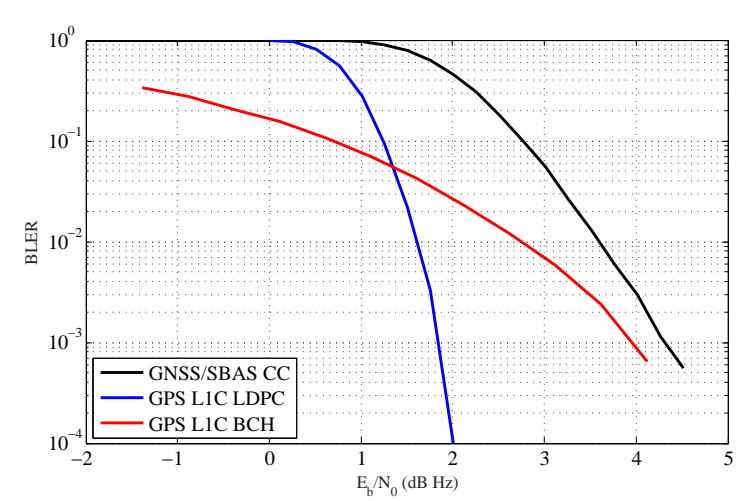

Fig. 3. Performance of channel codes used in GNSS/SBAS systems in terms of BLER vs $E_{b} / N_{0}$.

$\mathbf{p}_{\mathbf{2}}$, which are obtained as follows:

$$
\begin{aligned}
\phi & =-\mathbf{E} \mathbf{T}^{-1} \mathbf{B}+\mathbf{D} \\
\mathbf{p}_{\mathbf{1}}{ }^{t} & =-\phi^{-1}\left(-\mathbf{E} \mathbf{T}^{-1} \mathbf{A}+\mathbf{C}\right) \mathbf{u}^{t} \\
\mathbf{p}_{\mathbf{2}}{ }^{t} & =-\mathbf{T}^{-1}\left(\mathbf{A} \mathbf{u}^{t}+\mathbf{B p}_{\mathbf{1}}{ }^{t}\right) \\
\mathbf{c} & =\left[\begin{array}{ll}
\mathbf{u} & \mathbf{p}_{\mathbf{1}}
\end{array}\right]
\end{aligned}
$$

Decoding techniques are generally iterative and based on graphs algorithms and include, for example: the sum-product algorithm for general graph-based codes; maximum a posteriori probability (MAP) algorithms for trellis graph-based codes; the message passing algorithm for bipartite graph-based codes [42-44].

\section{Performance in AWGN}

To provide some context to the following sections, a brief Monte-Carlo simulation based performance evaluation of the three coding schemes is presented here. Error correction performance in the AWGN channel is provided in terms of BLER, where an entire block of decoded data is considered incorrect entirely if one or more bits are incorrect. In practical schemes, this is generally determined by examining the cyclic redundancy check (CRC). Recovery of the symbols can be performed in a number of ways, for example by examining the sign of the in-phase correlator channel, $\Re\left(Y_{n}\right)$, or via comparison of the data and pilot components of the composite signal, examining the sign of their dot-product, $\left(Y_{n}^{D} \cdot Y_{n}^{P}\right)$, where the superscripts $D$ and $P$ denote the data and pilot signal components, respectively. The first case requires that the receiver maintains phase-lock on the signal in order to perform data demodulation, while the second does not, and can provide data demodulation in the presence of high receiver dynamics or when the received signal is too weak to allow reliable phase tracking. Interestingly, when a long coherent integration period is applied to the pilot signal component, the demodulation performance of the second case converges to that of the first, despite the relaxed tracking requirement. For simplicity, in the analysis that follows it is assumed receiver is synchronized with the received signal and that the presence of thermal noise and interference only influences the data recovery.

Results are presented in two forms: in terms of energy-perbit, $E_{b} / N_{0}$, and in terms of carrier power, $C / N_{0}$. Expressing 


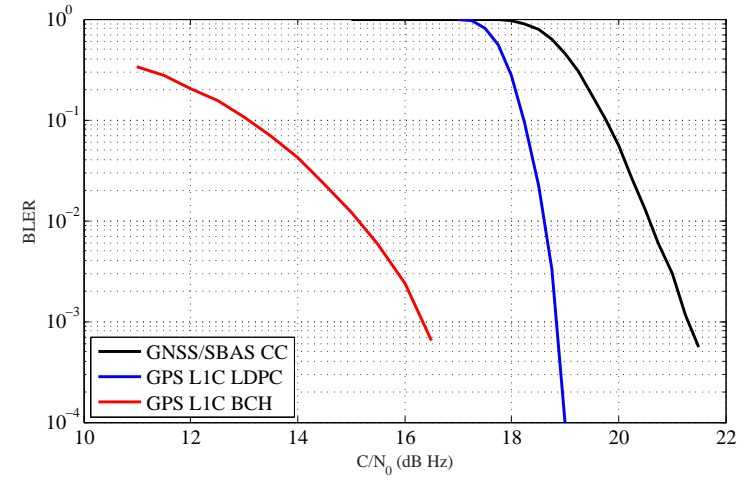

Fig. 4. Performance of channel codes used in GNSS/SBAS systems in terms of BLER vs $C / N_{0}$.

TABLE III

DEMODULATION THRESHOLDS FOR GNSS/SBAS

\begin{tabular}{|c|c|c|}
\hline \multirow{2}{*}{ FEC Scheme } & \multicolumn{2}{|c|}{ Required for BLER $=10^{-3}$} \\
\cline { 2 - 3 } & $E_{b} / N_{0}(\mathrm{~dB})$ & $C / N_{0}(\mathrm{~dB}-\mathrm{Hz})$ \\
\hline GNSS/SBAS CC & 4.31 & 21.30 \\
GPS L1C LDPC & 1.85 & 18.84 \\
GPS L1C BCH & 3.95 & 16.33 \\
\hline
\end{tabular}

performance in terms of $E_{b} / N_{0}$ provides some insight into the benefits, or coding gain, provided by a specific technique, without considering the coding rate; while expressing performance in terms of $C / N_{0}$ provides some more practical insight into the effective signal strength that might be required to attain useful receiver performance, not considering the efficiency of the coding scheme.

Results presented in Fig. 3 and 4 show the performance of the convolutional codes used by GPS, Galileo, EGNOS and WAAS against that of the BCH and LDPC codes used by the GPS L1C signal. To provide a fair comparison between LDPC and convolutional codes, a block length of 600 bits was considered, corresponding to one subframe of the GPS L1C signal.

The improved performance of the LDPC code is apparent in Fig. 3, providing a coding gain of approximately $2.5 \mathrm{~dB}$ with respect to the convolutional codes. For lower $E_{b} / N_{0}$ conditions, the $\mathrm{BCH}$ codes curve is the lowest BLER. However, for higher $E_{b} / N_{0}$ conditions, this margin reduces and ultimately the coding gain reduces to below that of the LDPC codes, suggesting that the benefits provided by the higher redundancy are, perhaps, limited by the shortness of the code. Thus rather than an assessment of the $\mathrm{BCH}$ performance itself, the curve characterizes the performance for this very specific design choice, where the protection of a very short message, of 9 bits, was required. Nonetheless, examining Fig. 4, the benefits of the $\mathrm{BCH}$ encoding of the GPS TOI are immediately apparent, providing reliably decoding to very low $C / N_{0}$ levels. Examining these figures it is possible to find approximate thresholds for a given demodulation BLER, of which those corresponding to a BLER of $10^{-3}$ are presented in Tab. III.

\section{Receiver Algorithms for Message Decoding UNDER JAMMING}

In this section we deal with the effect of jamming on the receiver message decoding, after tracking and symbol demodulation is performed. While it is in general difficult to identify the worst-case jamming signal for a given communication system, in the following we assume a generalized pulse jamming for several reasons. Firstly, it is general enough to include both pulse jamming as well as $\mathrm{CW}$ and additive noise jamming. Secondly, the generalized pulse jamming, which is detailed below, is not periodic and is therefore hardly predictable at the receiver. For these reasons, pulse jamming is often applied to assess the robustness of a communication system [46-48].

In more general terms, if one is free to design a communication system for maximum robustness against jamming, the worst-case jammer will be AWGN [49]. This is not the case for the GNSS systems considered here and we also would like to stress that any countermeasure against jamming which exploits features of channel coding naturally only can work if the jamming power is below a level which would affect the analog hardware, like e.g. driving amplifiers or the ADC into saturation or even destroy circuits.

When evaluating coding performance it is common to provide Bit-Error Rate (BER), BLER results for the AWGN channel with antipodal modulation, as we have provided in the previous section. However, in the presence of intentional interference or jamming such reference performance curves can be severely degraded. See for instance [50], where this evaluation is carried out for Deep-Space telecommand communication links. Although there are differences in the specific error correcting codes and the spreading involved, it provides a good analysis to understand the degradation the system may suffer in the presence of jamming and how it can be mitigated. In general terms: soft-decision decoding helps and strong codes, such as turbo codes and LPDC codes provide stronger protection. In [51] soft decision decoding is also proposed as an enhanced decoding approach in the presence of jamming where different estimators for the calculation of soft-input values are analyzed.

When evaluating anti-jamming capabilities of GNSS signals from a coding perspective we can distinguish two important coding components: first, the presence of a spreading sequence in the GNSS signal, which can be intepreted as a repetion code; second, the specific error correction code used by the different GNSS signal during encoding, as described in the previous section.

With respect to the former all spreading sequences used in GNSS systems share a common feature: they are pseudorandom sequences. These sequences are generated in various ways depending on the type of GNSS signal [52] including maximal-length sequences, Gold codes, memory codes, and Weil codes. In the following it will be shown that the most significant parameter in terms of anti-jamming capabilities is the sequence length.

With respect to the error correcting code the decoding performance greatly depends on the specific code selection 


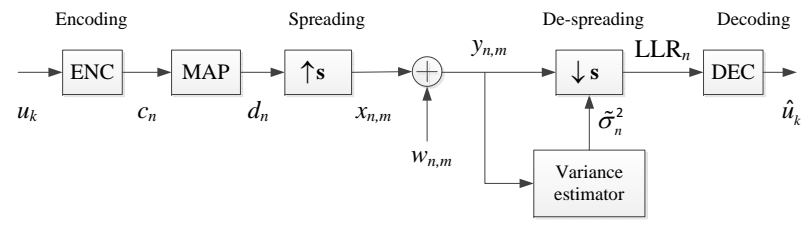

Fig. 5. Block diagram of a coded spread spectrum system.

and corresponding decoding algorithm. However, they have in common that most soft-decision decoding algorithms require the knowledge of the noise variance affecting each bit in the codeword. We shall remark that there are decoding algorithms like the max-log for LPDC decoding that do not explicitly requires the noise variance estimate, provided all coded bits are affected by the same noise variance. Otherwise decoding performance degrades. This is also the case of the Viterbi algorithm, where a normalization factor does not affect performance under the same previous assumptions. In the following, we show how the the spreading sequence can help on the estimation of the (equivalent) noise variance.

\section{A. System Model}

A simplified block diagram for the transmission and reception of the GNSS message is depicted in Fig. 5. The message is first encoded by one of the codes described in Sec. II, then the encoded bits are mapped to BPSK symbols and each symbol is multiplied by the spreading sequence. The receiver performs roughly the inverse steps in order to recover the original message. In this section, we will focus on the despreading operation and make use of soft decoding which operates on the probabilities of the coded bits.

1) Transmitter: At the transmitter, i.e. in the space segment, we represent the message as a binary vector $\mathbf{u}=$ $\left[u_{0}, u_{1}, \ldots, u_{K-1}\right]$ of $K$ bits. This message is encoded into a codeword $\mathbf{c}=\left[c_{0}, c_{1}, \ldots, c_{N_{\mathrm{c}-1}}\right]$ of length $N_{\mathrm{c}}>K$ and mapped to BPSK symbols $d_{n}=\mu\left(c_{n}\right) \in\{-1,1\}$, where we apply $\mu(0)=1$ and $\mu(1)=-1$. Denoting the spreading sequence by $\mathbf{s}=\left[s_{0}, s_{1}, \ldots, s_{N_{\mathrm{s}-1}}\right] \in\{-1,1\}^{N_{\mathrm{s}}}$, the transmitted symbols in base-band, also denoted as chips, are given by $x_{n, m}=d_{n} s_{m}$, with $n=0,1, \ldots, N_{\mathrm{c}}-1$ and $m=0,1, \ldots, N_{\mathrm{s}}-1$. The transmitted chip sequence per coded bit is therefore given by

$$
\mathbf{x}_{n}=d_{n} \cdot \mathbf{s}=\left[x_{n, 0}, x_{n, 1}, \ldots, x_{n, N_{\mathrm{s}}-1}\right], n=0, \ldots, N_{\mathrm{c}}-1
$$

and the number of chips per codeword is hence $N_{\mathrm{c}} N_{\mathrm{s}}$.

2) Channel and Interference Model: Among the different types of interference introduced in Sec. I-A, we focus on periodic pulsed interference, which is a representative case for the study of the impact of interference signal in terms of assessing the receiver decoding performance when the interference is highly uncorrelated with the actual transmitted signal.

In particular, we model the channel as the real-valued AWGN channel with noise variance, while we assume that the interference transmits impulses of length $N_{\mathrm{p}}$ chips with

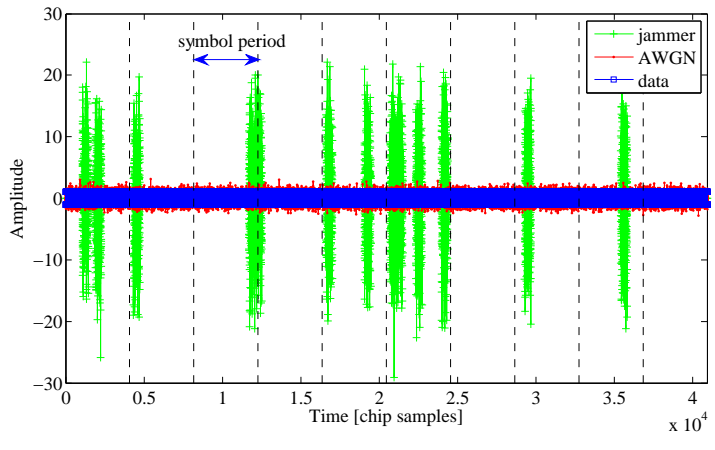

Fig. 6. Example interference impulses in relation to bit sequence.

probability $\rho$. This is a generalization of the periodic pulse interference with duty cycle $\rho$. Note that in general the interference pulses may not appear in regular intervals but can be randomly spaced which makes it hardly impossible for the receiver to detect the pulse length and periodicity of the interference. Hence, for the evaluation of the decoding performance, the interference signal is generated such that for each bit interval an interference pulse is generated with probability $\rho$ while the average power over the entire codeword is fixed.

With these assumptions, the received symbol sequence which corresponds to the coded bit $c_{n}$ is given by

$$
\mathbf{y}_{n}=d_{n} \cdot \mathbf{s}+\mathbf{w}_{n}, \quad y_{n, m}=d_{n} s_{m}+w_{n, m}
$$

where the noise is normal distributed with $w_{n, m} \sim$ $\mathcal{N}\left(0, \sigma_{n, m}^{2}\right)$ and

$$
\sigma_{n, m}^{2}= \begin{cases}\frac{N_{0}}{2 T_{c}} & \text { for clean chips } \\ \frac{N_{0}}{2 T_{c}}+\frac{J_{0}}{2 \rho T_{c}} & \text { for jammed chips }\end{cases}
$$

An illustrative example of the interference realization is shown in Fig. 6 where the interference, additive noise and data signal are depicted for the first chip samples of the transmitted codeword. The vertical discontinuous lines separate each coded symbol. In the example the pulsed interference duration is less than a symbol period, with some symbols affected with several interference pulses and others where the interference is not present at all.

\section{B. Soft Despreading}

The task of the despreading circuit at the receiver is to obtain the posterior probability of each bit, given the observed sequence $\mathbf{y}_{n}$. An equivalent metric is the log-likelihood ratio (LLR), which is defined as:

$$
\begin{aligned}
\operatorname{LLR}_{n} & \triangleq \ln \frac{P\left[c_{n}=0 \mid \mathbf{y}_{n}\right]}{P\left[c_{n}=1 \mid \mathbf{y}_{n}\right]}=\ln \frac{p\left(\mathbf{y}_{n} \mid d_{n}=1\right)}{p\left(\mathbf{y}_{n} \mid d_{n}=-1\right)} \\
& =\sum_{m=0}^{N_{\mathrm{s}}-1} \ln \frac{p\left(y_{n, m} \mid d_{n}=1\right)}{p\left(y_{n, m} \mid d_{n}=-1\right)}=\sum_{m=0}^{N_{\mathrm{s}}-1} \frac{2 s_{m}}{\sigma_{n, m}^{2}} y_{n, m}
\end{aligned}
$$

Note that this expression depends on the noise variance per chip which requires knowledge of the noise and jamming 
power per chip. This means that not only the jamming power but also the positions of the jamming impulses are required to compute the exact LLRs. Since in particular, we cannot assume the latter, we have to resort to a simpler metric.

In the case that all chips of the sequence $\mathbf{y}_{n}$ are affected by the same jamming pulse, we can express the noise and jamming power by the single parameter $\sigma_{n}^{2}$ and the expression for the LLR simplifies to

$$
\operatorname{LLR}_{n}=\frac{2}{\sigma_{n}^{2}} \sum_{m=0}^{N_{\mathrm{s}}-1} s_{m} y_{n, m}=\frac{2}{\sigma_{n}^{2}} \mathbf{s y}_{n}^{\mathrm{T}}
$$

1) Estimation of Jamming and Noise Power: Assuming a fixed noise variance per bit, the conditional pdf of the received sequence is

$$
p\left(\mathbf{y}_{n} \mid d_{n}, \sigma_{n}^{2}\right)=\left(\frac{1}{\sqrt{2 \pi \sigma_{n}^{2}}}\right)^{N_{\mathrm{s}}} \exp \left(-\frac{\left\|\mathbf{y}_{n}-d_{n} \mathbf{s}\right\|^{2}}{2 \sigma_{n}^{2}}\right)
$$

and the corresponding negative log-likelihood function

$$
\begin{aligned}
\mathcal{L}\left(d_{n}, \sigma_{n}^{2}\right) & \triangleq-\ln p\left(\mathbf{y}_{n} \mid d_{n}, \sigma_{n}^{2}\right) \\
& =\frac{N_{\mathrm{s}}}{2} \ln \left(2 \pi \sigma_{n}^{2}\right)+\frac{\left\|\mathbf{y}_{n}-d_{n} \mathbf{s}\right\|^{2}}{2 \sigma_{n}^{2}}
\end{aligned}
$$

which has the extremal points

$$
\begin{aligned}
& \frac{\partial \mathcal{L}\left(d_{n}, \sigma_{n}^{2}\right)}{\partial d}=0 \Rightarrow \hat{d}_{n}=\frac{\mathbf{s y}_{n}^{\mathrm{T}}}{N_{\mathrm{s}}} \\
& \frac{\partial \mathcal{L}\left(d_{n}, \sigma_{n}^{2}\right)}{\partial \sigma_{n}^{2}}=0 \Rightarrow \hat{\sigma}_{n}^{2}=\frac{1}{N_{\mathrm{s}}}\left\|\mathbf{y}_{n}-\hat{d}_{n} \mathbf{s}\right\|^{2}
\end{aligned}
$$

Notice that the product $\mathbf{s y}_{n}^{\mathrm{T}}$ corresponds to the de-spreading operation and thus $\hat{d}_{n}$ is precisely the prompt sample at the output of the correlators. This finally leads to the estimate of the noise and jamming power per bit, given by

$$
\hat{\sigma}_{n}^{2}=\frac{1}{N_{\mathrm{s}}}\left\|\mathbf{y}_{n}-\frac{\mathbf{s} \mathbf{y}_{n}^{\mathrm{T}}}{N_{\mathrm{s}}} \mathbf{s}\right\|^{2}
$$

As a reference to other receiver decoding schemes, we may consider:

1) hard despreading followed by hard-input Viterbi decoding,

$$
\hat{c}_{n}= \begin{cases}1 & \text { if } \mathbf{s y}_{n}^{\mathrm{T}}<0 \\ 0 & \text { otherwise }\end{cases}
$$

2) soft despreading with knowlegde of noise power only (this may represent the case where the background noise power is estimated - maybe on a different time-scale than per codeword - but the receiver is not aware of the interference)

$$
\operatorname{LLR}_{n}^{(1)}=\frac{4}{N_{0}} \mathbf{s y}_{n}^{\mathrm{T}}
$$

3) soft despreading with estimated noise and jamming power (the receiver observes the jamming power uniformly distributed over the codeword, although the actual jammer is not):

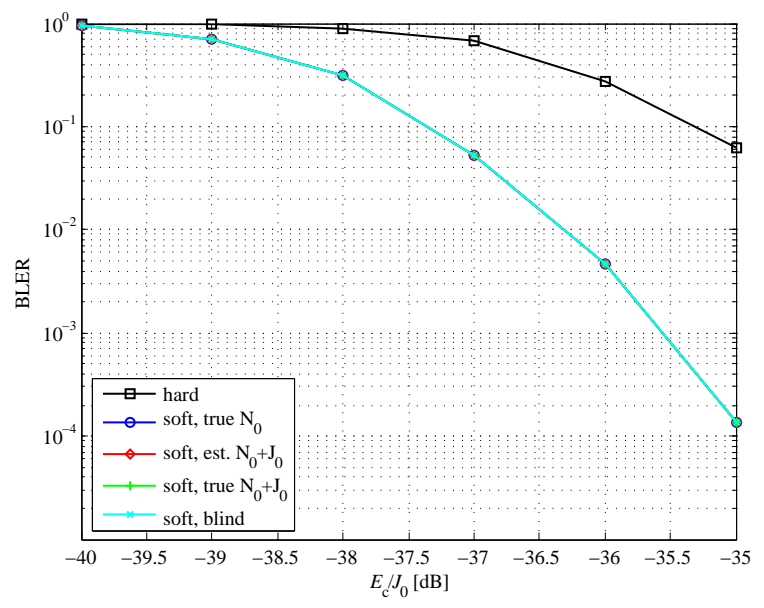

Fig. 7. BLER with interference for soft and hard decoding with $\rho=1$.

$$
\operatorname{LLR}_{n}^{(2)}=\frac{2}{\hat{\sigma}_{n}^{2}} \mathbf{s y}_{n}^{\mathrm{T}}
$$

4) soft despreading with knowledge of jamming power and positions (as a genie bound):

$$
\operatorname{LLR}_{n}^{(3)}=\sum_{m=0}^{N_{\mathrm{s}}-1} \frac{2 s_{m}}{\sigma_{n, m}^{2}} y_{n, m}
$$

5) soft despreading with no knowledge of signal to noise ratio (blind decoder):

$$
\operatorname{LLR}_{n}^{(5)}=\mathbf{s y}_{n}^{\mathrm{T}}
$$

\section{Simulation Results}

This section provides BLER and BER performance results for a reference scenario based on Galileo E1-B signal. For its GPS counterpart, L1 C/A (this signal implements no channel coding) simulation results are indicative of the performance degradation for different interference realizations.

The parameters used for the numerical assessment are specified in Table IV. Since the focus of this section is on interference impact on the decoding functionality we assume perfect synchronization and signal demodulation. In both scenarios the channel is generated according to (8) where the additive noise and interference samples are generated from two independent zero-mean normal distributions such that (9) is met.

Fig. 7 illustrates the performance gain of soft decoding versus hard decoding with LLR values for soft decoding computed as in (16) and (13). Taking as baseline a continuous interference with $\rho=1$, this is equivalent to raising the noise power. In the example, the E1-B signal is decoded with the Viterbi algorithm after de-spreading the received signal according to $\operatorname{LLR}_{n}^{(1)}, \operatorname{LLR}_{n}^{(2)}, \operatorname{LLR}_{n}^{(3)}, \operatorname{LLR}_{n}^{(4)}$ and $\operatorname{LLR}_{n}^{(5)}$, respectively. Simulations are carried out for a fixed signal to noise ratio $E_{c} / N_{0}=-10 \mathrm{~dB}$ (corresponding to a $C / N_{0}=50 \mathrm{~dB} / \mathrm{Hz}$ ) and variable $E_{c} / J_{0}$. As expected, when all coded symbols in a codeword are affected by the same noise variance, as it is the case when the interference is continuous 
TABLE IV

CODING AND SPREADING SCHEMES FOR REFERENCE SCENARIOS

\begin{tabular}{l|c|c} 
Simulation parameters & \multicolumn{2}{|c}{ System \& Signal } \\
\hline & L1 C/A & Galileo E1-B \\
\hline Coding & None & $1 / 2$-rate conv. \\
Message length & 300 bits & 114 bits +6 tail bits \\
Codeword length & 300 coded symbols & 240 coded symbols \\
Spreading sequence & 1023 Gold-code, repeated 20 times & 4092 random \\
Spreading gain & 20460 chips & 4092 chips \\
Chip rate & 1.023 Mcps & 1.023 Mcps \\
Symbol rate & 50 sps & 250 sps \\
Pulsed interference duration & $N_{p}=1 \%, 10 \%, 100 \%$ and $200 \%$ symbol duration & $N_{p}=1 \%, 10 \%, 100 \%$ and 200\% symbol duration \\
& $N_{p}=204,2046,20460$ and 4092 chips & $N_{p}=40,409,4092$ and 8184 chips
\end{tabular}

or has the same pulse length of a codeword, the Viterbi decoder is transparent to a scalar mismatch in the log-likelihood values. Hence, for all soft decoding realizations the BLER is the same with approximately $2 \mathrm{~dB}$ gain in terms of $E_{c} / J_{0}$ with respect to hard decoding, clearly showing the increase in robustness achieved with soft-decoding.

If we allow the interference to have lower duty-cycle then the decoding performance strongly depends on the knowledge of noise plus interference power at each coded symbol. This effect is clearly observed in Fig. 8 where the BLER at $E_{c} / N_{0}=-10 \mathrm{~dB}$ is depicted for $\rho=0.1$ and different pulse lengths. The pulse length is set to a percentage of the symbol duration, with the assumption that it corresponds to an integer number of chips. For the reference signal E1-B the interference pulse duration takes values $N_{p}=40,409$, 4092, and 8184 chips in the different simulation realizations. Note that the number of interference pulses in a codeword depends on the value of $\rho$ and $N_{p}$. That is, for the same interference power, and duty cycle $\rho$, the longer the pulse duration the less number of interference pulses are present in a codeword. The opposite occurs when the pulse length is reduced. In this case the number of symbols affected by interference increases. At each Monte-Carlo realization a single codeword is transmitted, with interference pulses positions being randomly generated.

Simulation results in Fig. 8 lead to the conclusion that having an estimate of the noise plus interference power always improves the decoder performance, even though the estimate is obtained at symbol level without actual knowledge of the pulsed interference position with chip granularity. While completely ignoring the presence of the interference may even result in worst performance than hard decoding, as it is apparent in the case of a interference affecting an entire symbol Fig. 8 (c) and (d). Similar results are obtained for other values of the duty cycle. Back to the E1-B signal, an example of the noise variance estimate compared to the actual noise + interference variance is depicted in Fig. 9 for two different values of pulsed interference length. Intuitively, for increasing values of $N_{p}$ the assumption made in (10) is more plausible and thus the results are closer to the optimal solution in (11).

It is important to remark that the gains of the proposed scheme against interference is practically independent of the actual spreading sequence. The differences are in the spreading gain, namely the spreading sequence length.

To conclude this section, we shall remark the significant

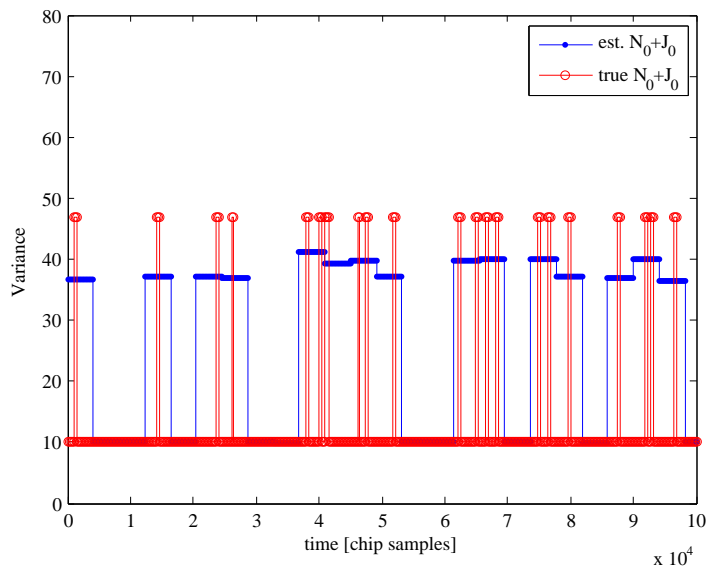

Fig. 9. Example of noise variance estimate for a pulsed interference with $\rho=0.1, N_{p}=409$ and $E_{\mathrm{c}} / J_{0}=-37 \mathrm{~dB}$

gains this simple, low complexity, noise plus interference variance estimator, resulting in $\operatorname{LLR}_{n}^{(2)}$, applied with soft channel decoding brings to GNSS interference environments. The estimator is aware of the presence of interference power but lacking the knowledge of where it appears.

\section{CODing SCHEMES For ANTI-SPOOFIng}

The coding schemes presented so far have focused on the correct extraction of a data sequence from a noise- or interference-corrupted set of received symbols. This process has been based upon the assumption that the underlying data is present, and is genuine. The coding schemes have sought only to discern the most likely value of the data sequence. In contrast, this section will examine the problem of determining whether or not a given data sequence, once extracted from the noise, is genuine and has originated at a GNSS satellite, or is counterfeit, and may have originated from a malicious transmitter.

Unfortunately, the need for secure GNSS for civilian users was not anticipated when current GNSSs were designed, and so a wide range of ad-hoc solutions have been proposed to satisfy this need without requiring changes to the satellite systems. These schemes have typically exploited the high entropy military GNSS signals, and observe them simultaneously from two locations, between which there is a trusted communication infrastructure. Although the concept is over two decades old $[53,54]$ it is a popular approach $[55,56]$. In this case, the 


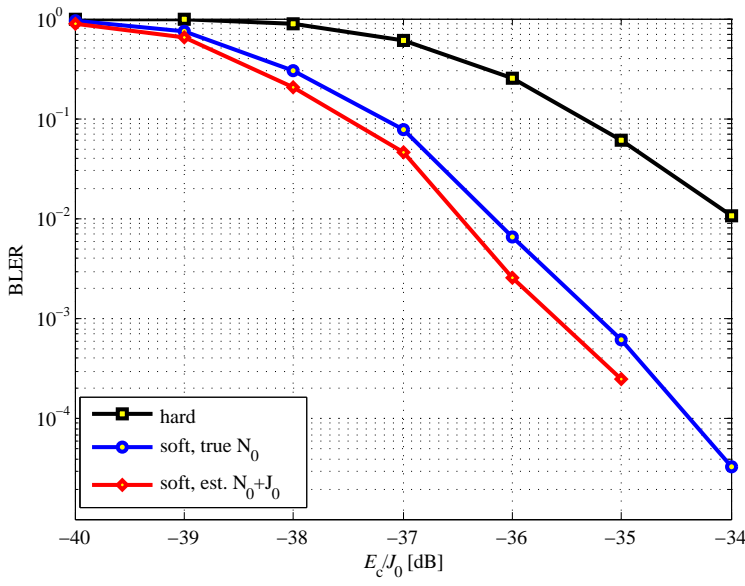

(a) $N_{p}=40$.

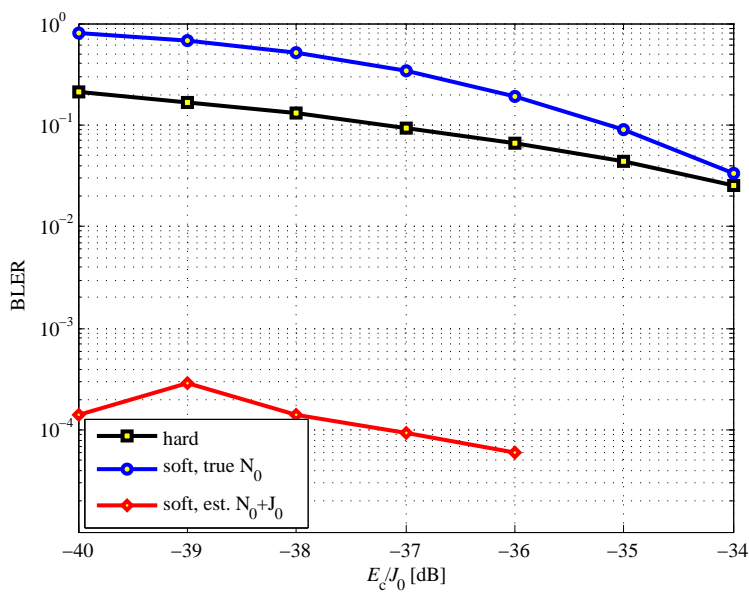

(c) $N_{p}=4092$.

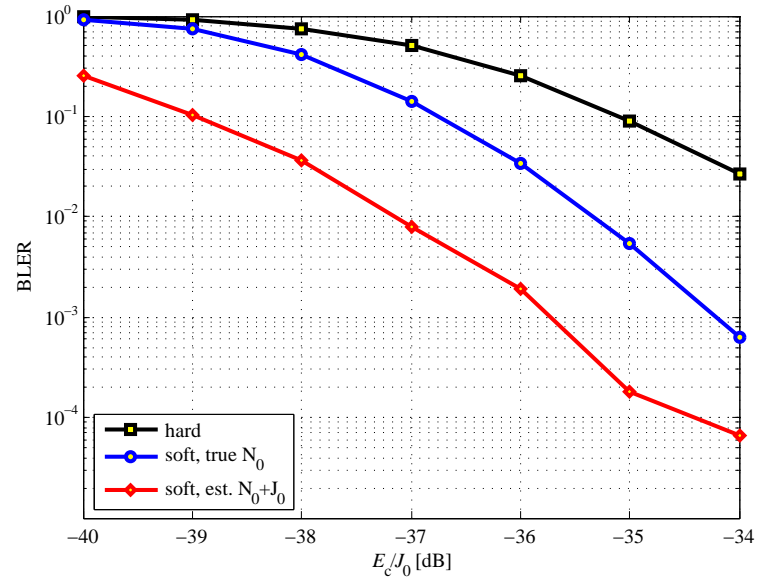

(b) $N_{p}=409$.

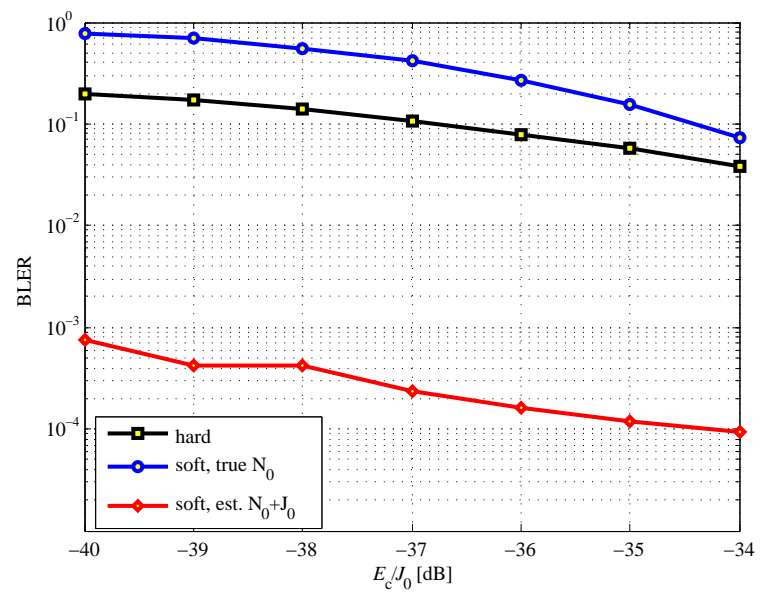

(d) $N_{p}=8184$.

Fig. 8. BLER for E1-B in the presence of pulsed interference with $\rho=0.1$ and $E_{\mathrm{c}} / N_{0}=-10 \mathrm{~dB}$.

security of one receiver is dependent upon the assumption that the other is secure, although this can be addressed via the use of an authorized receiver [57, 58].

In terms of system evolution there have been many proposals for schemes which can provide robust anti-spoofing, which can be categorized as either signal- or message-based. The signal-based variety generally aim to make the process described above more secure or more efficient [59-61], while the message based schemes typically introduce more classical cryptographic operations to the navigation message processing [60-65], as will be discussed further below.

\section{A. Requirements for Secure Navigation}

The vulnerability of a typical GNSS user stems from a variety of assumptions regarding the observed GNSS signal and its relation to physical space and time. A position computed by a naïve GNSS user makes two distinct assumptions which lead to its vulnerability: firstly, it is assumed that the data broadcast by the satellite and, subsequently used in the computation of a position, velocity and time solution (PVT), is genuine and, secondly, it is assumed that the observed time-of-arrival of the signal is strictly related to the geometric range between the user and the satellite. If either one of these assumptions are invalidated then so too is the associated PVT. An adversary might attempt to manipulate the PVT by preying on either or both the observed signal latency, termed the pseudorange, and its message content.

A user may wish to assure that the computed PVT is genuine, in the sense that its error is associated with nonmalicious factors, such as thermal noise signal-in-space errors and local propagation effects, rather than from intentional manipulation of the received signal by an adversary. To achieve this, two factors must be secured: the message content and the signal path.

The signal content encompasses the information payload of the signal, in a pure communication sense. In this case it includes information such as time-of-week, satellite ephemerides, clock corrections, atmospheric correction, satellite health and almanac data, etc. To ensure that the user can trust that satellite position and transmit-times gleaned from this data are genuine and correct, then the integrity of the data must be ensured. In a cryptographic sense, assuring data integrity is to assure the accuracy and consistency of the data, for which a large selection well established schemes exist. 
The signal path encompasses the propagation of the signal from the satellite to the user. In essence, time-of-arrival ranging is a channel estimation problem, whereby the length of the channel between the satellite and the user is measured via the broadcast GNSS signals. The user should be able to trust that the time-of-flight of the signal across this path is strictly related to the geometric range between the satellite and the user.

If a signal path to the user were to be described by the sequence of points: $p_{0}, p_{1}, \ldots, p_{N-1}, p_{N}$, where $p_{0}$ is the point of origin of the signal, and $p_{N}$ is the position of the user, then nominal navigation assumes that $p_{0}$ is the satellite and $N=1$. If a malicious adversary generates counterfeit signals and broadcasts them to the user, then $p_{0}$ is the adversary again, $N=1$. Alternatively, if this adversary intercepts genuine satellite signals, and manipulates them before rebroadcast to the user, then $p_{0}$ is the satellite, $p_{1}$ is the adversary, and $N=2$. A receiver may trust a PVT when a strict relationship between geometric range and signal path is ensured, which occurs when: $p_{0}$ is the satellite position, and $N=1$.

The first condition, that $p_{0}$ corresponds to the satellite position can be addressed as a problem of signal authenticity or data origin authentication. To ensure that the signal has originated from the system, via the satellite, it should contain some component which is infeasible for a malicious adversary to independently generate, but practical for a user to verify. Note that this requirement also implies that the signal be nonrepeating: having intercepted one signal period, it should not be possible to readily replicate signals not yet broadcast. Being a classic cryptography problem many appropriate schemes exist which can be directly applied in this case [60-64, 66, 67].

The second condition, that $N=1$, stipulates that the signal time-of-flight is entirely attributable to geometric range. This condition precludes the relaying of signals, received at one or more locations, towards a target receiver. Although there are many means by which a signal can be rendered difficult to manipulate and relay, unfortunately, given the constraints of the broadcast-only system, there appears to be no convenient coding-level means by which this condition can be rendered enforced to the same degree as the first.

Therefore, this section will focus only on the problem of ensuring that a user can readily assert that $p_{0}$ is the satellite. Facility for a user to verify this might be provided in the form of a navigation message authentication (NMA) scheme. Such a scheme may insert cryptographic content into available space in the signals navigation message which can be exploited by a user to verify the authenticity of the navigation message and to verify that it originated at the satellite.

\section{B. Digital Signatures}

The digital signature forms the basic building block of navigation message authentication schemes either by providing the NMA itself, or by facilitating other message authentication schemes, as discussed in Sec. IV-C. The purpose of a digital signature is to provide a means of guaranteeing who generated a given message and is a mechanism that relies on public-key cryptography. The basic principle is that a one party uses a private key to generate a signature for a given message, and a second party can use the corresponding public key to verify the signature. Verification of the signature provides both message integrity and data origin authentication.

When applied directly to the problem navigation message authentication, the signature is generated as a function of both the navigation data bits that are to be signed, and of the private key. Many well known signature schemes exist, for example RSA, DSA, ECDSA [64, 66, 67].

The system generates a public-private key pair: $\left\{K_{\text {priv }}, K_{\text {pub }}\right\}$, and shares $K_{\text {pub }}$ with the intended users. For a given block of navigation data, $\mathbf{u}$, a signature is generated as $\mathbf{s}=\mathbb{S}\left(K_{\text {priv }}, \mathbf{u}\right)$. If a GNSS signal were to employ a digital signature based NMA, then both $\mathbf{u}$ and $\mathbf{s}$ would be encoded to $\mathbf{c}$ and broadcast in the navigation message. At the receiver, the symbols would be recovered, decoded, and a verification operation would be conducted: $\mathbb{V}\left(K_{\text {pub }}, \mathbf{d}, \mathbf{s}\right)=$ true/false. Generally it is desirable to include some additional, frequently-changing data in the signature generation, to ensure that, even when the navigation data is repeating, successive signatures are different. Inclusion of the time-of-week, for example, can serve this purpose.

It is important to note that the verification operation relies on the correct recovery of the data and signature in their entirety. This places an additional burden on the receiver, over what is required for typical navigation applications. Under benign conditions, a receiver need only sporadically retrieve the navigation data, as ephemeris parameters have a long validity. To avail of trustworthy navigation, however, the receiver must maintain continuous data retrieval, as will be discussed in Sec. IV-D. This is especially critical for digital-signature based NMA, as public key cryptography is relatively inefficient. When considering ECDSA, to achieve $B$ bit security, (implying that a brute-force attack would require $2^{B}$ trials), a public key of $2 B$ bits must be used, and will require a signature of $4 B$ bits [66]. This fact is a motivator for the use of symmetric cryptographic schemes, where the relationship between key-size, signature and security is closer to unity.

\section{Message Authentication Codes}

A message authentication code (MAC) can be considered as a compact, cryptographically-secure fingerprint of a piece of data, and can offer an efficient way of verifying its authenticity. Many standard implementation exist, popular amongst them being the keyed-hash MAC, or HMAC, which is implemented using a cryptographic hash function. Such a hash function, $h(\cdot)$, operates on arbitrary length data streams, to producing a finite output, which is highly sensitive to changes in the input data.

If a GNSS signal were to employ a MAC-based NMA, then a symmetric key, $k_{i}$ would be used to generate a MAC $m_{i}$ as: $m_{i}=h\left(\mathbf{u}, k_{i}\right)$ and both $\mathbf{u}$ and $m_{i}$ would be encoded to navigation symbols and broadcast in the navigation message. At the receiver, the symbols would be recovered, decoded, and a verification operation would be conducted to assert that $h\left(\mathbf{u}, k_{i}\right)$ is equal to the received $m_{i}$. As $h(\cdot)$ preimageresistant, or difficult to invert, even with knowledge of $m_{i}$ and 
$\mathbf{u}$, the value of $k_{i}$ cannot be determined, thus only a holder of $k_{i}$ can generate a valid $m_{i}$. In the event that the system releases $k_{i}$ only after $\mathbf{u}$ and $m_{i}$ have been received by the user, then message authentication can be achieved.

A scheme known as TESLA provides this functionality [60-62], whereby message authentication codes are generated using a special series of symmetric keys, generated via:

$$
k_{i}=h^{N-i}\left(k_{0}\right), 0<i \leq N
$$

which defines a hash-chain, of length $N$. Once the root value $k_{0}$ has been shared between the system and the user, the authenticity of a $k_{i}$ can be asserted via (19). NMA can then be achieved by broadcasting, in sequence, the values $m_{i}$ then $\mathbf{u}$ and then $k_{i}$. A user will first verify $k_{i}$ and then use it to verify $m_{i}$, thereby authenticating the origin of $\mathbf{u}$. Noting that $N$ can be quite large, the remaining problem of securely sharing $k_{0}$ can be solved via the use of digital signatures, as described in Sec. IV-B, and broadcast only very rarely.

Such a scheme has a number of advantages over digital signatures. Firstly, the total data occupancy of $m_{i}$ and $k_{i}$ will be approximately half that of an ECDSA signature of equivalent security. Secondly, while a complete recovery of the signature is required for digital signatures, a user may decide to accept only a partial match in the verification of $m_{i}$, with the associated reduction in security, but gaining tolerance to a few bit errors.

\section{Denial Of Service}

For users who might avail of a GNSS NMA service, the concept of denial-of-service (DOS) can be reconsidered. Generally, DOS is considered to be the denial of the use of the GNSS signals for navigation purposes, typically performed by overwhelming the target receiver with an interference, such that the navigation signals may not be acquired or tracked. In such cases, it might be quite often obvious to the user that they the subject of a malicious attack. In fact, under certain circumstances, if the interference is sufficiently powerful, it is even possible to localize its source.

Considering an NMA user, if it is only necessary to disrupt the message authentication process, DOS might be achieved my simply ensuring that the message content, $s$ or $\left\{m_{i}, k_{i}\right\}$, is corrupted. This might allow a malicious adversary to broadcast a much lower interference power, with a very low dutycycle, while still disrupting receiver operation. This form of subtle interference, while disrupting the NMA service, might not induce any obvious disruption to the tracking and navigation functionality of the target receiver. Moreover, it might even allow the adversary to go undetected, leaving the target receiver unable to identify the cause of the authentication failure, or unable to localize the adversary.

To explore this problem, this section considers a hypothetical Galileo E1-B NMA scheme as a case study. It is assumed that the 'Reserved 1' field is used, which delivers 40 bits every two seconds. An adversary is assumed to broadcast pulsed interference, similar to that described in Sec. III, however rather than randomly timed interference pulses, they are arranged to maximize the disruption caused to the decoding of the NMA
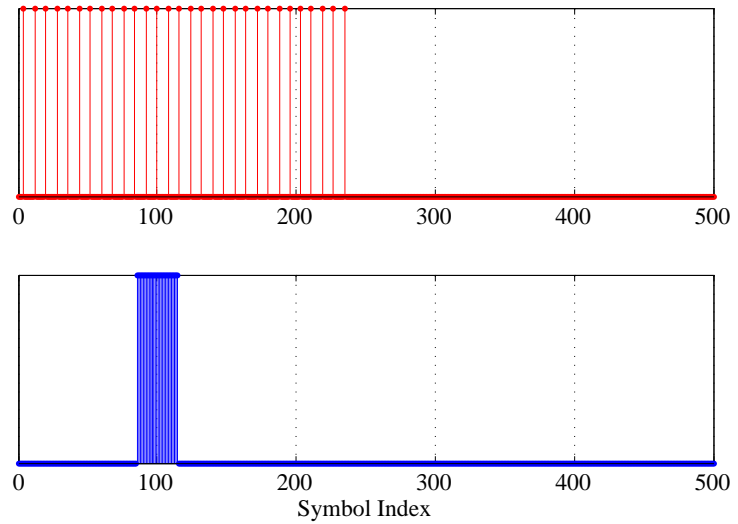

Fig. 10. Locations of 30 symbols that might be corrupted by an adversary (top), and their locations after de-interleaving (bottom), for one even and odd page of the Galileo E1-B message.

data. For simplicity it is assumed that one complete set of cryptographic data is broadcast over 15 consecutive 40-bit fields, offering one message authentication operation every 30seconds. To deny message authentication, an adversary might attempt to corrupt one of these 40-bit portions. As a case study, it is assumed that the adversary targets the first 40-bit block of each 30 -second period, denoted hereafter by $\mathbf{u}_{\mathrm{NMA}}^{(1)}$.

In the previous section, as both the thermal noise and interference were assumed to be uncorrelated over time, the block interleaving of the navigation message, $\mathbf{u}$, has been neglected, here, however, it becomes an important aspect. Block interleaving is a process of re-arranging the codeword symbols before broadcast, such that adjacent symbols are dispersed when broadcast. Once received, these symbols are deinterleaved before decoding. Burst of errors, spanning multiple symbols, during the signal transmission will appear as sparse errors after the symbols are de-interleaved, which can be more easily corrected by the coding scheme. Block interleaving can be represented as filling a matrix column-wise with $\mathbf{c}$, taking it's transpose, and extracting the data, again column-wise. For Galileo E1-B a $30 \times 8$ interleaving matrix is used.

When targeting a specific portion of the received navigation message, it is in the adversarys interest to corrupt the symbols which are related to these bits. For a $1 / r$-rate convolutional encoder of constraint-length $L$, then the encoding of $k$ consecutive bits will influence a total of $r \times(k+L)$ symbols (94 symbols for E1-B signal).

Once synchronized with a GNSS signal, a malicious adversary may then broadcast interference at specific intervals such that, once de-interleaved, a continuous portion of the symbols related to $\mathbf{u}_{\mathrm{NMA}}^{(1)}$ is corrupted, thereby maximizing the likelihood of corrupting $\mathbf{u}_{\mathrm{NMA}}^{(1)}$. This is depicted in Fig. 10, where every eighth symbol is corrupted for a total of 30 symbols.

When striving to maximize the likelihood of corrupting $\mathbf{u}_{\mathrm{NMA}}^{(1)}$, or maximizing the corresponding authentication error rate (AER), the adversary may adjust three variables: the pulse duration; the instantaneous interference power; and the number of symbols to corrupt. To negate the effectiveness of 


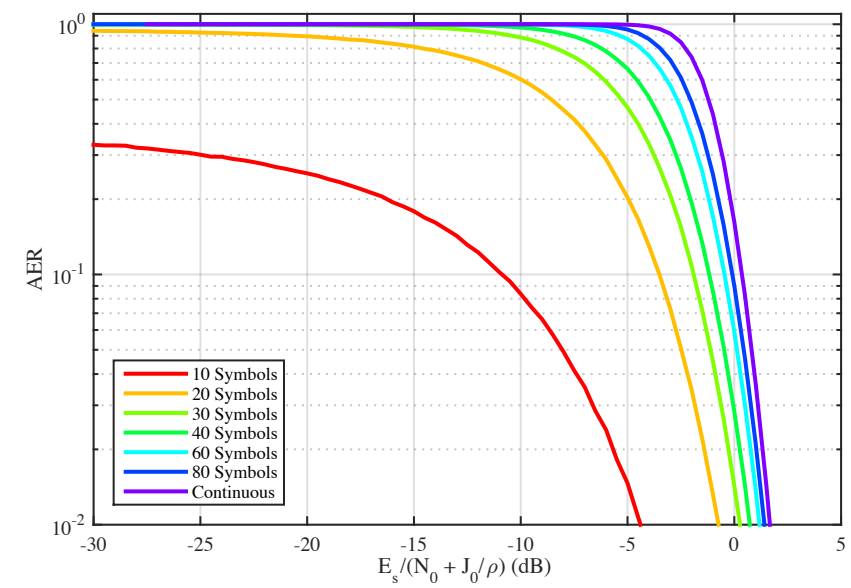

Fig. 11. AER versus instantaneous interference strength $E_{s} /\left(N_{0}+J_{0} / \rho\right)$, using hard-decision Viterbi decoding, considering various numbers of symbol corruptions as well as the continuous interference case.

pule-blanking, it is clear that the pulse duration should be set equal to, and aligned with, the received symbols, such that $N_{p}=4096$, as defined in Sec. III. The number of symbols to corrupt should be as low as possible, while still overwhelming the FEC decoder, so at least longer than the constraint-length, and thus in the range: $[8,94]$. The number of symbols to corrupt effectively defines an average interference duty-cycle, $\rho$, as defined in Sec. III, over the entire 30 second authentication period. Note that in this case it takes on an extremely small value: $0.0013 \leq \rho \leq 0.0125$.

The influence of the instantaneous interference power, and number of symbol corruptions on the AER is explored in Fig. 11 for a hard-decision Viterbi decoder. As would be expected, for a given instantaneous interference power, as the number of symbols is increased, the AER increases. Is can be seen that corrupting as few as 80 symbols, equivalent to $\rho=0.01$, yields an AER very close to the continuous interference case. It seems that a calculated and synchronized interference attack can achieve similar service disruption to the continuous interference case, with approximately $1 \%$ of the average power.

Further insight into this can be gained by rescaling Fig. 11 by the $\rho$, to present the AER as a function of average interference power, as shown in Fig. 12. It is evident that corrupting as few as 30 to 80 symbols offers a very efficient means for an adversary to deny the NMA service. To provoke an AER of 0.9 in the target receiver, then an adversary might broadcast a synchronized corruption of 40 symbols, requiring an average power approximately $20 \mathrm{~dB}$ lower than if a continuous interference were used. In this case, the target receiver observes an $E_{s} / N_{0}$ of approximately $16 \mathrm{~dB}$, or a $C / N_{0}$ of $40 \mathrm{dBHz}$, which is not alarmingly low under nominal conditions. Similarly, if an AER of 0.99 is to be provoked, 60 symbols might be targeted, providing an average interference power reduction of approximately $19 \mathrm{~dB}$ relative to a continuous interference.

Fig. 13 depicts similar results for soft-decision Viterbi decoding. In this case, the conservative assumption is made that the receiver has a perfect estimate of the $\left(N_{0}+J_{0} / \rho\right)$

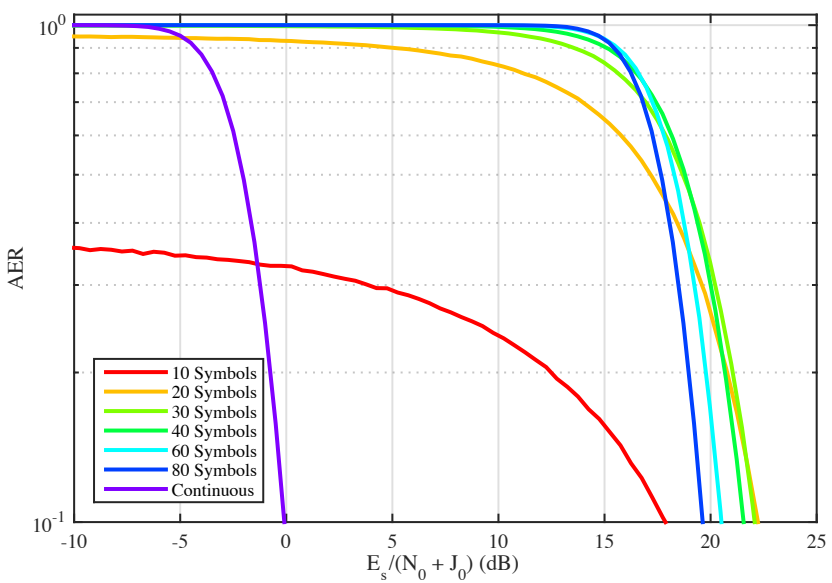

Fig. 12. AER versus average interference strength $E_{s} /\left(N_{0}+J_{0}\right)$ using hard-decision Viterbi decoding, considering various numbers of symbol corruptions as well as the continuous interference case.

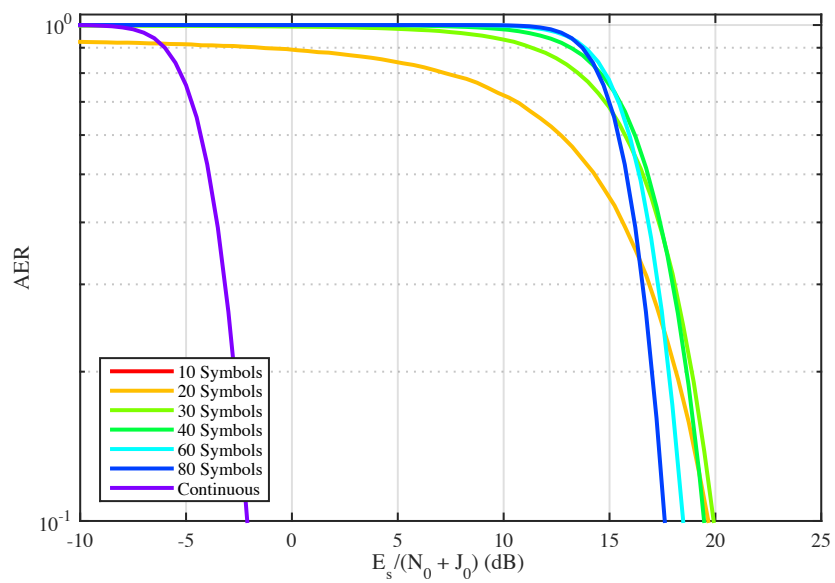

Fig. 13. AER versus average interference strength $E_{s} /\left(N_{0}+J_{0}\right)$ using softdecision Viterbi decoding with perfect $\left(N_{0}+J_{0} / \rho\right)$ estimation, considering various numbers of symbol corruptions as well as the continuous interference case.

value for each symbol. As would be expected, the curves are generally offset by approximately $2 \mathrm{~dB}$, relative to the hard-decision case. However, in this case, for fewer than 20 symbol corruptions, soft-decoding provides near perfect error correction, and so the 10-symbol curve has been omitted. Again, it appears that a margin of approximately $19 \mathrm{~dB}$ exists between the continuous interference and the synchronizedpulsed interference cases.

These results seem to expose a potential vulnerability of a future NMA user, that an adversary might be capable of disrupting normal receiver operation by broadcasting a GNSSsynchronized interference with an extremely low average power. As the duty-cycle is so low, and the pulses are so well dispersed, it is also likely that this interference may not induce a noticeable malfunction of any other receiver component. As $C / N_{0}$ estimation typically employs smoothing of one second or longer [8], it is likely that it will be unaffected. Code and carrier tracking systems will likely operate on the pilot signal E1-C, and may use a coherent integration period of longer than one-symbol. As the bandwidth of the tracking loops is 
relatively narrow, of the order of Hertz for the code and some tens of Hertz for the carrier, it is likely that code and carrier observations will provide no obvious indication either.

It is somewhat unfortunate, that the use of a blockinterleaver appears to work in favor of the adversary in this particular case. If it were necessary to corrupt long runs of adjacent symbols, it might be easier to become aware of adversarial conditions. Nonetheless, it is clear that a receiver which intends to exploit an NMA service should host some form of sample-level monitoring system to detect such forms of subtle-interference.

\section{Conclusions}

Modern GNSS signals employ a diverse selection of errorcorrecting codes which provide robust data reception, not only in the classical AWGN or fading channels, but also in the face of an adversary who is broadcasting a malicious interference. This article evidenced that this performance can be further improved via receiver enhancements, such as soft-decision decoding. Its implementation requires minimal receiver modifications and can be embedded using the basic building blocks of a GNSS receiver. Results show that a soft-decoding scheme provides robustness to a generic type of pulsed jamming interference, in contrast to hard-decoding approaches.

As the use of GNSS penetrates ever more in critical applications, the need for robust and reliable GNSS is growing. Since the adoption of some signal-borne cryptographic schemes seems to be a viable defense against counterfeit signals, the availability of the navigation message is central to securing GNSS. Results show that a well calibrated attack can readily disrupt a naïve navigation message authentication scheme. As such, it is clear that these schemes should not only be robust in a security sense, but also robust against noise and interference.

Unfortunately, although the improved receiver technology can deliver substantial gains, such improved technology in the hands of an adversary might be equally powerful. It is possible that future GNSS signals may require even more powerful encoding and that receivers may be required to adapt to the threat of a more sophisticated adversary.

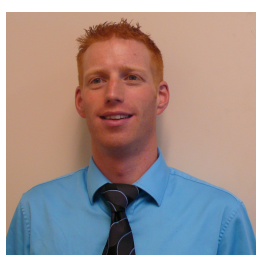

James T. Curran (M'06)received a B.E. in Electrical \& Electronic Engineering in 2006 and a Ph.D. in Telecommunications in 2010, from the Department of Electrical Engineering, University College Cork, Ireland. He worked as a senior research engineer with the PLAN Group in the University of Calgary from 2011 in the area of software-defined radio for GNSS and signal-processing techniques for interference mitigation. He is currently a grant-holder, researching radio-navigation and GNSS vulnerability at the Joint Research Center (JRC) of the European Commission (EC), Italy. His main research interests are signal processing, information theory, cryptography and software defined radio for GNSS.

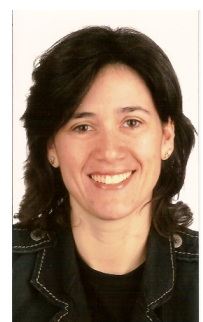

Monica Navarro is a Senior Researcher at the Centre Tecnològic de Telecomunicacions de Catalunya within the Communication Systems Division. She received the MSc degree in Telecommunications Engineering from Universitat Politècnica de Catalunya in 1997 and the $\mathrm{PhD}$ degree in Telecommunications from the Institute for Telecommunications Research (ITR), University of South Australia, in 2002. From Oct. 1997 to Dec. 1998 she was a Research Assistant at the Department of Signal Theory and Communications at the UPC, where she worked on the development of fractal shape multiband antennas for wireless cellular communications systems. She has also been part-time lecturer at the Universitat Pompeu Fabra, Barcelona. Her primary areas of interest are on digital communications and signal processing, particularly on iterative information processing, adaptive transmissions and coding techniques, signal processing for synchronization, estimation and detection theory with applications to radio communications systems, including wireless mobile communications, deepspace communications, wireless sensor networks, and positioning applications.

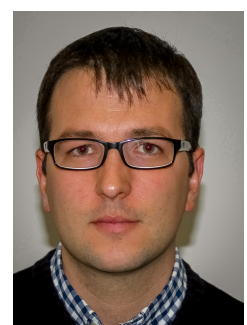

Marco Anghileri is navigation systems engineer at Airbus Defence \& Space, where he currently leads various $R \& D$ activities on the evolution of navigation systems and signals. In the past, he was lead systems engineer at IFEN GmbH and research associate at the Institute of Space Technology and Space Applications of the Universität der Bundeswehr in Munich. His main research interests include navigation signal and message design and performance, receiver algorithms as well as compatibility and interoperability of RNSS. From 2009 to 2013 he was personally involved in the European GNSS Programme, supporting the European Commission and the European GNSS Agency in different working groups, where he served as EU member state technical expert. He received his M.Sc. in Electrical Engineering from the Politecnico di Milano, Italy in 2006.

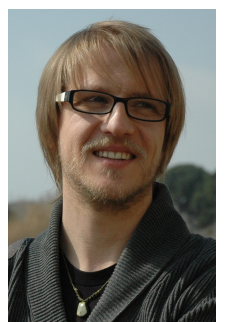

Pau Closas (S'04-M'10-SM'13) received the M.Sc. and $\mathrm{Ph} . \mathrm{D}$. in Electrical Engineering from the Universitat Politècnica de Catalunya (UPC) in 2003 and 2009, respectively. He also holds a M.Sc. degree in Advanced Mathematics and Mathematical Engineering from UPC since 2014. In 2003, he joined the Department of Signal Theory and Communications, UPC, as a Research Assistant. During 2008 he was Research Visitor at the Stony Brook University (SBU), New York, USA. In September 2009 he joined the CTTC, where he currently holds a position as a Senior Researcher and Head of the Statistical Inference for Communications and Positioning Department. He has many years of experience in projects funded by the European Commission, Spanish and Catalan Governments, as well as the European Space Agency in both technical and managerial duties. His primary areas of interest include statistical and array signal processing, estimation and detection theory, Bayesian filtering, robustness analysis, and game theory, with applications to positioning systems, wireless communications, and mathematical biology. Pau Closas is Senior Member of IEEE, ION, and EURASIP. He was involved in the organizing committees of EUSIPCO'11, IEEE IMWS'11, IEEE RFID-TA'11, European Wireless'14, IEEE SSP'16, and IEEE ICASSP'20 conferences. He is the recipient of the EURASIP Best PhD Thesis Award 2014 and the $9^{\text {th }}$ Duran Farell Award for Technology Research, both in recognition to his contributions to the field of signal processing for GNSS. 


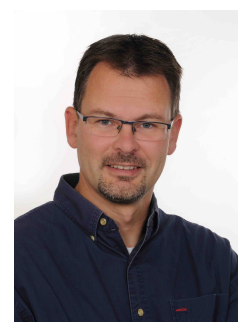

Stephan Pfletschinger has received his Master and Ph.D. degree from the University of Stuttgart (Germany) in 1997 and 2003. From 1997 to 2003 he was with the Institute of Telecommunications at the University of Stuttgart as a research and teaching assistant, working on multicarrier modulation and digital TV. For his Ph.D. thesis he received the Rudolf-Urtel and the Anton-und-Klara-Röser award. In March 2003 he joined the Centre Tecnològic de Telecomunicacions de Catalunya (CTTC) in Barcelona, where actively contributed to numerous European and industrial research projects on wireless communications, including systems beyond $3 \mathrm{G}$, radio access technologies and non-binary coding technologies. From 2007 to 2012 he was involved in teaching undergraduate courses on communications theory as an Associate Professor at the Universitat Pompeu Fabra. In July 2015, he joined the German Aerospace Center (DLR) in Oberpfaffenhofen to work on train-to-train and industrial communications. In March 2016, he has been appointed Professor for Communication Technologies at the University of Applied Sciences Offenburg. His primary research interests include iterative receiver algorithms, network coding, cross-layer link adaptation and non-binary information processing techniques.

\section{ACKNOWLEDGMENTS}

This work has been partially supported by the Spanish Ministry of Economy and Competitiveness through project TEC2015-69868-C2-2-R (ADVENTURE) and by the Government of Catalonia under Grant 2014-SGR-1567.

\section{REFERENCES}

[1] R. J. Landry and A. Renard, "Analysis of potential interference sources and assessment of present solutions for GPS/GNSS receivers," in Proc. of the 4th International Conference on Integrated Navigation Systems, St. Petersburg, May 1997.

[2] V. Calmettes, F. Pradeilles, and M. Bousquet, "Study and comparison of interference mitigation techniques for GPS receiver," in Proc. of the 14th International Technical Meeting of the Satellite Division of The Institute of Navigation, Sept 2001, pp. 957-968.

[3] R. H. Mitch, R. C. Dougherty, M. L. Psiaki, S. P. Powell, B. W. O'Hanlon, J. A. Bhatti, and T. E. Humphreys, "Signal characteristics of civil GPS jammers," in Proc. 24rd Int. Tech. Meeting Sat. Div. Inst. Navig., Portland, OR, Sep. 2011, pp. 1907-1919.

[4] H. Kuusniemi, E. Airos, M. Zahidul, H. Bhuiyan, and T. Kroger, "Effect of GNSS jammers on consumer grade satellite navigation receivers," in Proc. of the European Navigation Conference (ENC), Gdanks, Poland, April 2012, pp. 1-14.

[5] C. Gunther, "A Survey of Spoofing and CounterMeasures," NAVIGATION, Journal of The Institute of Navigation, vol. 61, no. 3, pp. 159-177, Fall 2014.

[6] J. Betz, "Effect of narrowband interference on GPS code tracking accuracy," in In Proceedings of the Institute of Navigation National Technical Meeting (ION NTM 2000), Anaheim, CA, Jan. 2000.

[7] —_, "Effect of partial-band interference on receiver estimation of C/NO: Theory," in In Proceedings of the Institute of Navigation National Technical Meeting (ION NTM 2001), Long Beach, CA, Jan. 2001.

[8] E. D. Kaplan and C. J. Hegarty, Eds., Understanding GPS. Principles and Applications, 2nd ed., ser. Mobile
Communications Series. Norwood, MA: Artech House, 2006.

[9] B. Motella, S. Savasta, D. Margaria, and F. Dovis, "Method for Assessing the Interference Impact on GNSS Receivers," Aerospace and Electronic Systems, IEEE Transactions on, vol. 47, no. 2, pp. 1416-1432, April 2011.

[10] J. Jang, M. Paonni, and B. Eissfeller, "CW Interference Effects on Tracking Performance of GNSS Receivers," Aerospace and Electronic Systems, IEEE Transactions on, vol. 48, no. 1, pp. 243-258, Jan 2012.

[11] R. L. Fante and J. Vaccaro, "Wideband cancellation of interference in a GPS receive array," Aerospace and Electronic Systems, IEEE Transactions on, vol. 36, no. 2, pp. 549-564, 2000.

[12] G. Seco-Granados, J. Fernandez-Rubio, C. FernándezPrades et al., "ML estimator and hybrid beamformer for multipath and interference mitigation in GNSS receivers," Signal Processing, IEEE Transactions on, vol. 53, no. 3, pp. 1194-1208, 2005.

[13] M. G. Amin and W. Sun, "A novel interference suppression scheme for global navigation satellite systems using antenna array," Selected Areas in Communications, IEEE Journal on, vol. 23, no. 5, pp. 999-1012, 2005.

[14] D. S. De Lorenzo, J. Rife, P. Enge, and D. M. Akos, "Navigation accuracy and interference rejection for an adaptive GPS antenna array," in Proceedings of the Institute of Navigation, vol. 2006, 2006, pp. 763-773.

[15] A. Konovaltsev, D. S. De Lorenzo, A. Hornbostel, and P. Enge, "Mitigation of continuous and pulsed radio interference with GNSS antenna arrays," Institute of Navigation GNSS, 2008.

[16] P. Closas and C. Fernández-Prades, "A statistical multipath detector for antenna array based GNSS receivers," Wireless Communications, IEEE Transactions on, vol. 10, no. 3, pp. 916-929, 2011.

[17] S. Daneshmand, A. Jafarnia-Jahromi, A. Broumandan, and G. Lachapelle, "A low-complexity gps anti-spoofing method using a multi-antenna mrray," in Proceedings of the 25th International Technical Meeting of The Satellite Division of the Institute of Navigation, 2012, pp. 12331243.

[18] J. Arribas, C. Fernandez-Prades, and P. Closas, “Antenna array based GNSS signal acquisition for interference mitigation," Aerospace and Electronic Systems, IEEE Transactions on, vol. 49, no. 1, pp. 223-243, 2013.

[19] J. Arribas, P. Closas, C. Fernández-Prades, M. Cuntz, M. Meurer, and A. Konovaltsev, "Advances in the theory and implementation of GNSS antenna array receivers," Microwave and Millimeter Wave Circuits and Systems: Emerging Design, Technologies, and Applications, pp. 227-273, 2013.

[20] J. T. Curran, M. Bavaro, and J. Fortuny, "Analog and digital nulling techniques for multi-element antennas in gnss receivers," in Proceedings of the 28th International Technical Meeting of The Satellite Division of the Institute of Navigation, September 2015, pp. 3249 - 3261.

[21] M. Abdizadeh, J. T. Curran, and G. Lachapelle, "Quan- 
tization Effects in GNSS Receivers in The Presence of Interference," in Proc. Intl. Tech. Meeting Inst. Navig., Newport Beach, CA, Jan. 2012, pp. 742-779.

[22] S. Gunawardena, J. Dickman, and M. A. Cosgrove, "Systems and methods for adaptive sample quantization," US Patent US 8923414 B2, Dec. 30, 2014.

[23] F. Bastide, D. Akos, C. Macabiau, and B. Roturier, "Automatic Gain Control (AGC) as an Interference Assessment Tool," in Proc. 16th Int. Tech. Meeting Sat. Div. Inst. Navig., Porland, OR, Sep. 2003, pp. 2042-2053.

[24] T. Pany, Navigation signal processing for GNSS software receivers. Norwood, MA: Artech House, 2010, ch. 6. Sample Processing.

[25] Y. R. Chien, "Design of GPS anti-jamming systems using adaptive notch filters," IEEE Systems Journal, vol. 9, no. 2, pp. 451-460, June 2015.

[26] Science Applications International Corporation, Interface Specification IS-GPS-200 Revision E. Navstar GPS Space Segment/Navigation User Interfaces, El Segundo, CA, June 2010.

[27] European Union, European GNSS (Galileo) Open Service. Signal In Space Interface Control Document. Ref: OS SIS ICD, Issue 1.2, Brussels, Belgium, Nov. 2015.

[28] M. Anghilieri, M. Paonni, D. Fontanella, and B. Eissfeller, "Assesing GNSS data message performance. a new approach," in Inside GNSS, April 2013.

[29] M. Pini, M. Fantino, A. Cavaleri, S. Ugazio, and L. L. Presti, "Signal Quality Monitoring Applied to Spoofing Detection," in Proceedings of the 24th International Technical Meeting of The Satellite Division of the Institute of Navigation, Portland, OR, Sep. 2011, pp. 18881896.

[30] A. Jafarnia-Jahromi, A. Broumandan, J. Nielsen, , and G. Lachapelle, "GPS vulnerability to spoofing threats and a review of antispoofing techniques," International Journal of Navigation and Observation, vol. 12, pp. 116, May 2012.

[31] M. L. Psiaki and S. P. P. amd B. W. O'Hanlon, “GNSS Spoofing Detection using High-Frequency Antenna Motion and Carrier-Phase Data," in Proceedings of the 26th International Technical Meeting of The Satellite Division of the Institute of Navigation, Sep 2013, pp. 2949-2991.

[32] K. D. Wesson, B. L. Evans, and T. E. Humphreys, "A combined symmetric difference and power monitoring GNSS anti-spoofing techniques," in Proc. of the IEEE Global Conference on Signal and Information Processing, Austin, 2005, p. 217220.

[33] S. Fantinato, G. Gamba, M. Anghileri, and R. Ioannides, "The Spoofing Estimating Delay Lock Loop," in Satellite Navigation Technologies and European Workshop on GNSS Signals and Signal Processing, (NAVITEC), Tampa, FL, 3-5 Dec. 2014.

[34] E. G. Manfredini, B. Motella, and F. Dovis, "Signal quality monitoring for discrimination between spoofing and environmental effects inducing correlation," in Proc. of the 28th International Technical Meeting of the Satellite Division of the ION, Tampa, FL, 10-13 Sept. 2015.

[35] P. Closas, J. Arribas, and C. Fernandez, "Spoofing detec- tion by a reduced acquisition process," in Proceedings of the 2016 International Technical Meeting of The Institute of Navigation, January 2016.

[36] P. Swaszek, R. Hartnett, and K. Seals, "GNSS Spoof Detection using Independent Range Information," in Proceedings of the 2016 International Technical Meeting of The Institute of Navigation, January 2016.

[37] J. W. Betz and D. B. Goldstein, "Candidate designs for an additional civil signal in GPS spectral bands," Proceedings of ION-GNSS-2002, vol. IT-13, pp. 622631, September 2002.

[38] B. Uchoa-Filho, R. Demo Souza, C. Pimentel, and M.C. Lin, "Generalized punctured convolutional codes," Communications Letters, IEEE, vol. 9, no. 12, pp. 1070 1072, Dec 2005.

[39] A. J. Viterbi, "Error bounds for convolutional codes and an asymptotically optimum decoding algorithm," Information Theory, IEEE Transactions on, vol. IT-13, pp. 260-269, April 1967.

[40] G. D. Forney, "The Viterbi Algorithm," Proceedings of the IEEE, vol. 61, pp. 268-278, March 1973.

[41] ARINC Engineering Services, "Navstar GPS Space Segment/User Segment L1C Interfaces," Draft IS-GPS-800, Rev. B, 2011.

[42] J. Moura, J. Lu, and H. Zhang, "Structured low-density parity-check codes," Signal Processing Magazine, IEEE, vol. 21, no. 1, pp. 42-55, Jan 2004.

[43] R. G. Gallager, "Low Density Parity Check Codes," Monograph, M.I.T. Press, 1963.

[44] D. MacKay, "Good error-correcting codes based on very sparse matrices," Information Theory, IEEE Transactions on, vol. 45, no. 2, pp. 399-431, Mar 1999.

[45] T. Richardson, M. Shokrollahi, and R. Urbanke, "Design of capacity-approaching irregular low-density paritycheck codes," Information Theory, IEEE Transactions on, vol. 47, no. 2, pp. 619-637, 2001.

[46] M. Baldi, M. Bianchi, F. Chiaraluce, R. Garello, N. Maturo, I. Sanchez, and S. Cioni, "Advanced Coding Schemes against Jamming in Telecommand Links," in Military Communications Conference, MILCOM 2013 2013 IEEE, Nov 2013, pp. 1220-1226.

[47] D. Torrieri, "The Performance of Five Different Metrics Against Pulsed Jamming," Communications, IEEE Transactions on, vol. 34, no. 2, pp. 200-204, Feb 1986.

[48] R.-H. Dou and L. Milstein, "Coded Error Probability for DS Spread-Spectrum Systems with Periodic Pulsed Multiple-Tone Interference," Communications, IEEE Transactions on, vol. 33, no. 10, pp. 1126-1132, Oct 1985.

[49] R. Blahut, Modem Theory: An Introduction to Telecommunications. Cambridge, UK New York: Cambridge University Press, 2010.

[50] M. Baldi, M. Bianchi, F. Chiaraluce, R. Garello, S. Cioni, and I. A. Sanchez, "Channel Coding Schemes Protection Againts Jamming for Direct Sequence Spread Sprectrum Telecommand Links," in Proc. of the 6th Int'l Worshop on Tracking, Telemetry and Command Systems for Space Applications(TTC 2013), ESA-ESOC, Darmstadt, Ger- 
many, 10-13 Sept. 2013, pp. 2044-2053.

[51] S. Pfletschinger, X. Mestre, M. Navarro, and P. Closas, "Soft Decoding for DS-SS Satellite Links Under Pulse Jamming," in In Proceedings of 6th ESA International Workshop on Tracking, Telemetry and Command Systems for Space Applications, Darmstadt, Germany, Sep. 2013.

[52] G. Hein, J. A. Ávila-Rodríguez, and S. Wallner, "The Galileo code and others," Inside GNSS, vol. 1, no. 6, pp. 62-74, Sep 2006.

[53] M. Barnard, "Vehicle location system," Sep. 4 1991, european Patent Register. EP19,910,200,359.

[54] P. MacDoran, M. Mathews, F. Ziel, K. Gold, S. Anderson, M. Coffey, and D. Denning, "Method and apparatus for authenticating the location of remote users of networked computing systems," May 1998, u.S. Patent 5757916.

[55] S. Lo, D. D. Lorenzo, P. Enge, D. Akos, and P. Bradley, "Signal Authentication: A Secure Civil GNSS for Today," in Inside GNSS, October 2009, pp. 30-39.

[56] M. Psiaki, B. O’Hanlon, J. Bhatti, D. Shepard, and T. Humphreys, "GPS Spoofing Detection via DualReceiver Correlation of Military Signals," Aerospace and Electronic Systems, IEEE Transactions on, vol. 49, no. 4, pp. 2250-2267, October 2013.

[57] A. Rugamer, M. Stahl, I. Lukcin, and G. Rohmer, "Privacy protected localization and authentication of georeferenced measurements using Galileo PRS," in Position, Location and Navigation Symposium - PLANS 2014, 2014 IEEE/ION, May 2014, pp. 478-486.

[58] M. Turner, A. Chambers, E. Mak, L. E. Aguado, B. Wales, and M. Dumville, "PROSPA: Open Service Authentication," in Proceedings of the 26th International Technical Meeting of The Satellite Division of the Institute of Navigation, September 2013, pp. 2992-2996.

[59] L. Scott, "Anti-Spoofing and Authenticated Signal Architectures for Civil Navigation Systems," in Proceedings of the 16th International Technical Meeting of the Satellite Division of The Institute of Navigation, September 2003, pp. 1543-1552.

[60] O. Pozzobon, L. Canzian, M. Danieletto, and A. Dalla Chiara, "Anti-spoofing and open GNSS signal authentication with signal authentication sequences," in Satellite Navigation Technologies and European Workshop on GNSS Signals and Signal Processing (NAVITEC), Dec 2010, pp. 1-6.

[61] J. T. Curran and M. Paonni, "Securing GNSS: An End-toEnd Feasibility Study for the Galileo Open Service," in Proceedings of the 27th International Technical Meeting of The Satellite Division of the Institute of Navigation, September 2014, pp. 2828 - 2842.

[62] C. Wullems, O. Pozzobon, and K. Kubik, "Signal Authentication and Integrity Schemes for Next Generation Global Navigation Satellite Systems," in Proceedings of the European Navigation Conference, April 2005, pp. 110.

[63] A. Kerns, K. Wesson, and T. Humphreys, "A blueprint for civil GPS navigation message authentication," in Position, Location and Navigation Symposium - PLANS
2014, 2014 IEEE/ION, May 2014, pp. 262-269.

[64] K. Wesson, M. Rothlisberger, and T. Humphreys, "Practical Cryptographic Civil GPS Signal Authentication," NAVIGATION, Journal of The Institute of Navigation, vol. 59, no. 3, pp. 177-193, Fall 2012.

[65] J. T. Curran, M. Paonni, and J. Bishop, "Securing the Open-Service: A Candidate Navigation Message Authentication Scheme for Galileo E1 OS," in European Navigation Conference - ENC 2014, April 2014, pp. 115.

[66] C. Paar, Understanding Cryptography: A Textbook for Students and Practitioners. Berlin London: Springer, 2009.

[67] A. J. Menezes, of Applied Cryptography. Boca Raton: CRC Press, 1997. 